\title{
Characterization of Inflammatory Gene Expression and Chemotaxis of Macrophages Expressing Guanylin and Guanylyl Cyclase-C
}

\author{
Kazuya Hasegawa, Sayaka Akieda-Asai, Yukari Date* \\ Frontier Science Research Center, University of Miyazaki, Miyazaki, Japan
}

Email address:

dateyuka@med.miyazaki-u.ac.jp (Y. Date)

To cite this article:

Kazuya Hasegawa, Sayaka Akieda-Asai, Yukari Date. Characterization of Inflammatory Gene Expression and Chemotaxis of Macrophages Expressing Guanylin and Guanylyl Cyclase-C. American Journal of Life Sciences. Special Issue: Biology and Medicine of Peptide and Steroid Hormones. Vol. 3, No. 3-2, 2015, pp. 43-47. doi: 10.11648/j.ajls.s.2015030302.18

\begin{abstract}
Depending upon the environment, macrophages can show at least two different phenotypes, including the inflammatory (M1) phenotype and the anti-inflammatory (M2) phenotype. CD11c-positive M1 macrophages produce proinflammatory cytokines such as interleukin (IL) $1 \beta$, IL-6, tumor necrosis factor $\alpha$, and monocyte chemoattractant protein (MCP) 1, which are linked to the development of obesity-associated insulin resistance. Recently, we showed that double-transgenic (dTg) rats overexpressing guanylin $(\mathrm{Gn})$ and its receptor, guanylyl cyclase-C (GC-C), specifically in macrophages did not become obese even when fed a high-fat diet. In the present study, to characterize macrophages expressing Gn and GC-C (i.e., Gn/GC-C macrophages), we analyzed the expression of the M1 and M2 markers of peritoneal macrophages isolated from dTg and wild type (WT) rats. We also examined the chemotaxis of these macrophages after incubation with MCP-1 or fatty acids. The expression of CD11c, an M1 macrophage marker were expressed at a significantly lower level in the peritoneal macrophages of dTg rats than in those of wild-type (WT) rats. In addition, the expression of IL-1, MCP-1 and chemokine receptor 2 were expressed at a significantly lower level in the peritoneal macrophages of dTg rats than in those of WT rats. On the other hand, there were no significant differences in the expression of M2 markers such as CD206, IL10, and arginine 1 between $\mathrm{dTg}$ and WT rats. We also found that the chemotaxis of $\mathrm{Gn} / \mathrm{GC}-\mathrm{C}$ macrophages incubated with fatty acids significantly increases compared to the macrophages of WT rats. Our results suggest that the low levels of proinflammatory cytokines and M1 markers in $\mathrm{Gn} / \mathrm{GC}-\mathrm{C}$ macrophages at least in part contribute to the anti-obese phenotype of $\mathrm{Gn} / \mathrm{GC}-\mathrm{C}$ Tg rats. In addition, the accelerated chemotaxis of Gn/GC-C macrophages in response to fatty acids suggests that these macrophages can uniquely react to excess fatty acids.
\end{abstract}

Keywords: Chemotaxis, Fatty Acids, Proinflammatory Cytokines

\section{Introduction}

Circulating monocytes differentiate into macrophages, which can migrate into tissues under steady state conditions or in response to inflammation [1,2]. Macrophages are highly heterogeneous cells found in nearly all tissues of the body [3-5] and can exhibit at least two different phenotypes: the classically activated M1 phenotype and the alternatively activated M2 phenotype [6,7]. CD11c-positive M1 macrophages are characterized by high expression of proinflammatory cytokines such as interleukin (IL) $1 \beta$, IL-6, tumor necrosis factor $\alpha$ (TNF- $\alpha)$, and monocyte chemoattractant protein (MCP) 1 [8], which are involved in inflammation in tissues [9-12]. In contrast, mannose receptor
CD206-positive M2 macrophages are characterized by high expression of anti-inflammatory cytokines such as IL-10 $[13,14]$ and arginase (Arg) $1[15,16]$, which are involved in the repair or remodeling of tissues $[4,9,17]$. Macrophages are able to modify their phenotype according to their environment. Obesity is strongly associated with a chronic inflammatory state and macrophage infiltration into adipose tissue has been observed in obese mice $[18,19]$. Most tissue macrophages that infiltrate into the adipose tissue of obese mice are CD11c-positive M1 macrophages responsible for the progress of adipose tissue inflammation [20,21].

Guanylin (Gn) and its receptor, guanylyl cyclase-C (GC-C), are known to be present mainly in the intestine and function to maintain homeostasis of body fluid [22]. Recently, we showed 
that double-transgenic (dTg) rats overexpressing $\mathrm{Gn}$ and GC-C specifically in macrophages did not become obese even when the rats were fed a high-fat diet [23]. This implies that the $\mathrm{Gn} / \mathrm{GC}-\mathrm{C}$ system in macrophages regulates obesity. From this finding, we expected that $\mathrm{Gn} / \mathrm{GC}-\mathrm{C}-$-expressing macrophages might have a unique balance between the opposing M1 and M2 phenotypes.

Here, to characterize $\mathrm{Gn} / \mathrm{GC}-\mathrm{C}$-expressing macrophages, we isolated peritoneal macrophages from $\mathrm{Gn} / \mathrm{GC}-\mathrm{C} \mathrm{dTg}$ rats, and analyzed the expression of M1 and M2 macrophage markers. We also examined the chemotaxis of these macrophages after incubation with MCP-1 or fatty acids.

\section{Materials and Methods}

\subsection{Animals}

The Gn/GC-C dTg rat has been described previously [23]. Male wild-type (WT) and dTg rats were housed individually in plastic cages in a room at constant temperature and under a 12:12-h light:dark cycle (lights on, 08:00 to 20:00). The rats were allowed ad libitum access to food and water throughout the study. All rats received standard laboratory chow (3.4 $\mathrm{kcal} / \mathrm{g})$ containing $4.6 \%(\mathrm{w} / \mathrm{w})$ fat, $51 \%(\mathrm{w} / \mathrm{w})$ carbohydrate, and $25 \%(w / w)$ protein (CE-2; CLEA Japan, Fuji, Shizuoka, Japan). All procedures were performed in accordance with the Japanese Physiological Society's guidelines for animal care. This protocol was approved by the Ethics Review Committee for Animal Experimentation of the Faculty of Medicine, University of Miyazaki.

\subsection{Isolation of Peritoneal Macrophages}

WT and dTg rats were injected intraperitoneally with 2 $\mathrm{ml} / \mathrm{kg}$ body weight of $4 \%$ thioglycollate. Exudate cells were collected by washing the peritoneal cavity with $0.9 \%(\mathrm{w} / \mathrm{v})$ saline 4 days after injection. The cells were incubated for $3 \mathrm{~h}$ at $37^{\circ} \mathrm{C}$ in a humidified $5 \%(\mathrm{v} / \mathrm{v}) \mathrm{CO}_{2}$ incubator, and adherent cells were used as peritoneal macrophages [24]. The macrophages were processed for RNA extraction or chemotaxis analysis as described below.

\subsection{Quantitative Pcr}

Total RNA from the peritoneal macrophages of WT and $\mathrm{dTg}$ rats ( $\mathrm{n}=5$ or 7 per group) was extracted with an RNeasy Plus Micro Kit (Qiagen, Hilden, Germany). First-strand cDNA was synthesized from the total RNA by using an Superscript III First-Strand Synthesis System kit (Invitrogen, Carlsbad, CA, USA). The resultant cDNA samples were subjected to quantitative PCR for CD11c, IL-1 $\beta$, IL-6, TNF- $\alpha$, MCP-1, chemokine receptor (CCR) 2, CD206, IL-10, and Arg1 by using a LightCycler system (Roche Diagnostics GmbH, Mannheim, Germany) with the SYBR Premix Ex Taq mix system (Takara Bio Inc., Shiga, Japan) and the primer sets listed in Table 1. The relative abundance of each reaction product was normalized to the level of ribosomal protein $36 \mathrm{~B} 4$ mRNA.

\subsection{Chemotaxis}

Chemotaxis of peritoneal macrophages obtained from WT and $\mathrm{dTg}$ rats ( $\mathrm{n}=5$ or 7 per group) was assessed with the CytoSelect 96-well Cell Migration Assay kit (Cell Biolabs, San Diego, CA, USA) according to the manufacturer's protocol. In brief, $5 \times 105$ macrophages in serum free RPMI 1640 medium (Nacalai Tesque, Kyoto, Japan) were placed in the upper chamber on 8-mm polycarbonate nucleopore filters, and the bottom well was filled with RPMI 1640 medium containing $2 \%(\mathrm{w} / \mathrm{v})$ fatty acid-free BSA and $200 \mathrm{ng} / \mathrm{ml}$ MCP-1 or $600 \mu \mathrm{M}$ fatty acid mixture $(120 \mu \mathrm{M}$ each of arachidonic, lyric, linoleic, myristic, and oleic acid). After incubation at $37{ }^{\circ} \mathrm{C}$ for $24 \mathrm{~h}$, macrophages that had migrated into the lower chamber were lysed in the presence of CyQUANT GR dye, and their fluorescent intensity was measured by using an excitation wavelength of $485 \mathrm{~nm}$, an emission wavelength of $538 \mathrm{~nm}$, and an emission cutoff of 515 $\mathrm{nm}$, with the use of a Flexstation Fluorometer (Molecular Devices, Sunnyvale, CA, USA).

Table 1. Primers used for quantitative PCR.

\begin{tabular}{|c|c|c|}
\hline Gene & Sequence & \\
\hline \multirow{2}{*}{ 36B4 } & Sense & TCATTGTGGGAGCAGACAATGTG \\
\hline & Antisense & AGGTCCTCCTTGGTGAACACAAA \\
\hline \multirow{2}{*}{ CD11c } & Sense & AAGCCCAAGTGTTCCTTCG \\
\hline & Antisense & CACATGAGGTGCAGGGAGT \\
\hline \multirow{2}{*}{ IL-1 $\beta$} & Sense & TGTGATGAAAGACGGCACAC \\
\hline & Antisense & СTTCTTCTTTGGGTATTGTTTGG \\
\hline \multirow{2}{*}{ IL-6 } & Sense & CACTTCACAAGTCGGAGGCTT \\
\hline & Antisense & TCTGACAGTGCATCATCGCTG \\
\hline \multirow{2}{*}{ TNF- $\alpha$} & Sense & TGAACTTCGGGGTGATCG \\
\hline & Antisense & GGGCTTGTCACTCGAGTTTT \\
\hline \multirow{2}{*}{ MCP-1 } & Sense & TGTAGCATCCACGTGCTGTC \\
\hline & Antisense & CCGACTCATTGGGATCATCT \\
\hline \multirow{2}{*}{ CCR2 } & Sense & AAGAAGTATCCAAGAGCTTGATGAG \\
\hline & Antisense & TCACCATCATCATAGTCATACGG \\
\hline \multirow{2}{*}{ CD206 } & Sense & TGGGTTTGCTGAAGAAGAGAA \\
\hline & Antisense & CATGTGATAAGTGACAAATGCTTG \\
\hline \multirow{2}{*}{ IL-10 } & Sense & AGTGGAGCAGGTGAAGAATGA \\
\hline & Antisense & TCATGGCCTTGTAGACACCTT \\
\hline \multirow{2}{*}{ Arg1 } & Sense & CCGCAGCATTAAGGAAAGC \\
\hline & Antisense & CCCGTGGTCTCTCACATTG \\
\hline
\end{tabular}

\subsection{Statistical Analysis}

We compared groups of data by using Student's t-tests (GraphPad Prism 5, GraphPad, San Diego, CA, USA). P values less than 0.05 were considered significant (two-tailed tests). Data are reported as means $\pm \mathrm{SEM}$.

\section{Results and Disucussion}

In this study, to characterize $\mathrm{Gn} / \mathrm{GC}-\mathrm{C}$-expressing macrophages, we investigated the expression of inflammatory cytokines in peritoneal macrophages isolated from $\mathrm{Gn} / \mathrm{GC}-\mathrm{C} \mathrm{dTg}$ and WT rats. CD11c-positive M1 macrophages produce proinflammatory cytokines, such as IL-1 $\beta$, IL-6, TNF- $\alpha$, and MCP-1, which are linked to the development of obesity-associated insulin resistance [25-28]. Our results showed that the mRNA expression levels of 
CD11c, IL-1, and MCP-1 were significantly lower in the peritoneal macrophages of $\mathrm{dTg}$ rats than in those of WT rats (Fig. 1). IL-6 and TNF- $\alpha$ showed the same trend as the other proinflammatory cytokines, but the differences in levels between $\mathrm{dTg}$ and WT rats were not statistically significant. The expression of CCR2, the receptor of MCP-1 [29], was also lower in the peritoneal macrophages of $\mathrm{dTg}$ rats than in those of WT rats (Fig. 1). In contrast, the M2 macrophage markers (CD206, IL-10, and Arg1) did not differ significantly between $\mathrm{dTg}$ rats and WT rats (Fig. 1). These results showed that $\mathrm{Gn} / \mathrm{GC}-\mathrm{C}$-expressing macrophages derived from $\mathrm{dTg}$ rats express low levels of proinflammatory cytokines under steady state conditions.

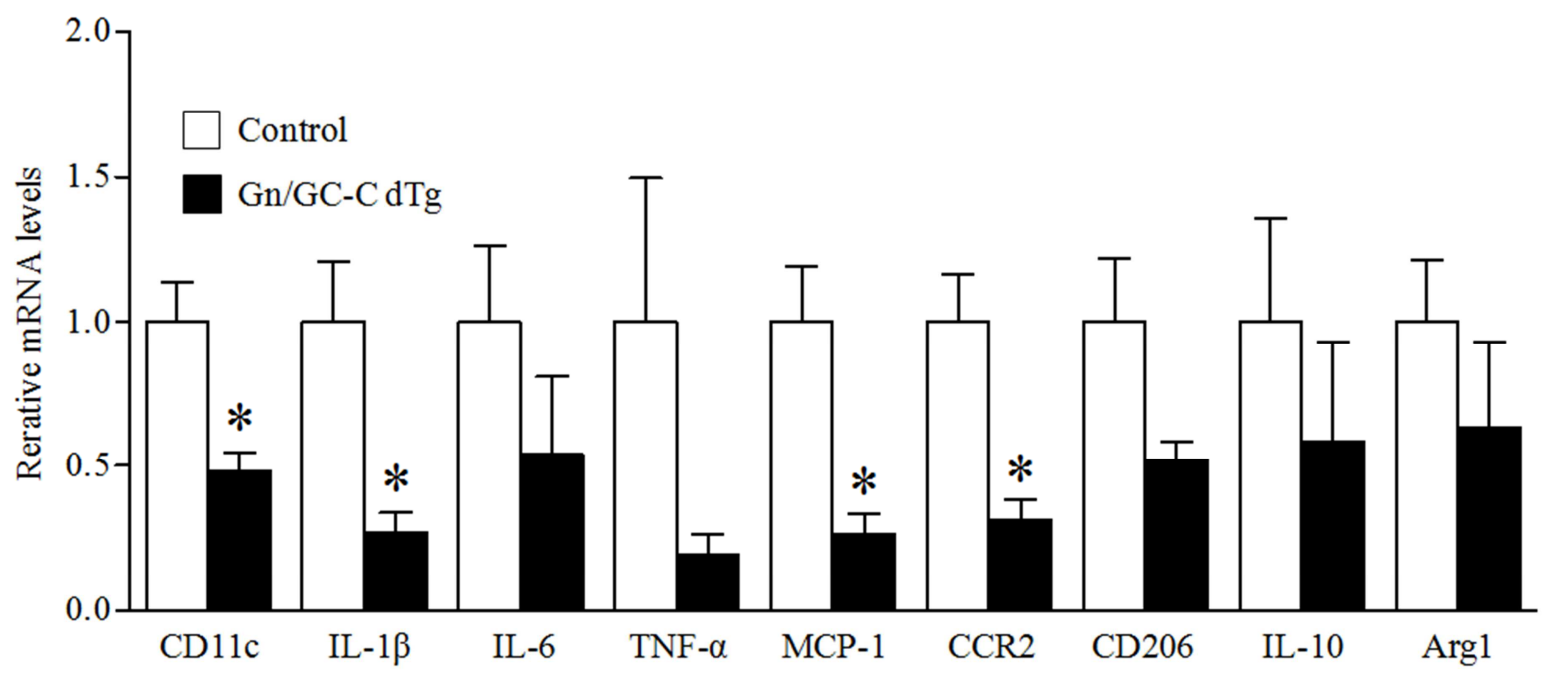

Fig. 1. Characterization of the markers of inflammatory (M1) and anti-inflammatory (M2) phenotypes in peritoneal macrophages from wild-type (WT) and $\mathrm{Gn} / G C-C d T g$ rats. Gene expression of M1 macrophage markers: CD11c, interleukin (IL) 1 $\beta$, IL-6, tumor necrosis factor $\alpha$ (TNF $\alpha$ ), monocyte chemoattractant protein (MCP) 1, and chemokine receptor (CCR) 2. Gene expression of M2 macrophage markers: CD206, IL-10, and arginase (Arg) 1. Data were normalized to $36 B 4 \mathrm{mRNA}$ levels and are presented as means $\pm S E M(n=5$ or 7 per group). $*, P<0.05$ compared with WT rats.

Recently, we showed that $\mathrm{dTg}$ rats overexpressing Gn and GC-C specifically in macrophages did not become obese even after ingesting a high- fat diet [23]. Taken together, these findings suggest that $\mathrm{Gn} / \mathrm{GC}-\mathrm{C}-$-expressing macrophages have unique "anti-inflammatory" characteristics under conditions in which inflammation would be expected to be induced (e.g., a high fat diet). To clarify how $\mathrm{Gn} / \mathrm{GC}-\mathrm{C}$ system regulates inflammatory state, further investigation will be required.

Fatty acids [30,31] and proinflammatory cytokines including MCP-1 [32,33] are known to recruit monocytes and macrophages to sites of inflammation in adipose tissue. Therefore, we examined the chemotaxis of peritoneal macrophages isolated from $\mathrm{Gn} / \mathrm{GC}-\mathrm{C} \mathrm{dTg}$ and WT rats after incubation with fatty acids or MCP-1. The chemotaxis of macrophages after incubation with MCP-1 did not differ between $\mathrm{dTg}$ rats and WT rats. However, the chemotaxis of macrophages after incubation with fatty acids was significantly induced in these macrophages of dTg rats than in those of WT rats (Fig. 2). Recently, we found that the mRNA expression of Gn and GC-C in a cell line significantly increased when the cells were incubated with fatty acids (data not shown). This finding indicates that the expressions of Gn and GC-C in macrophages may be induced at least in part by an excess of fatty acids. Together, fatty acids may act as not only a functional trigger of $\mathrm{Gn} / \mathrm{GC}-\mathrm{C}$ macrophages but also an inducer to promote the expressions of Gn and GC-C in macrophages.

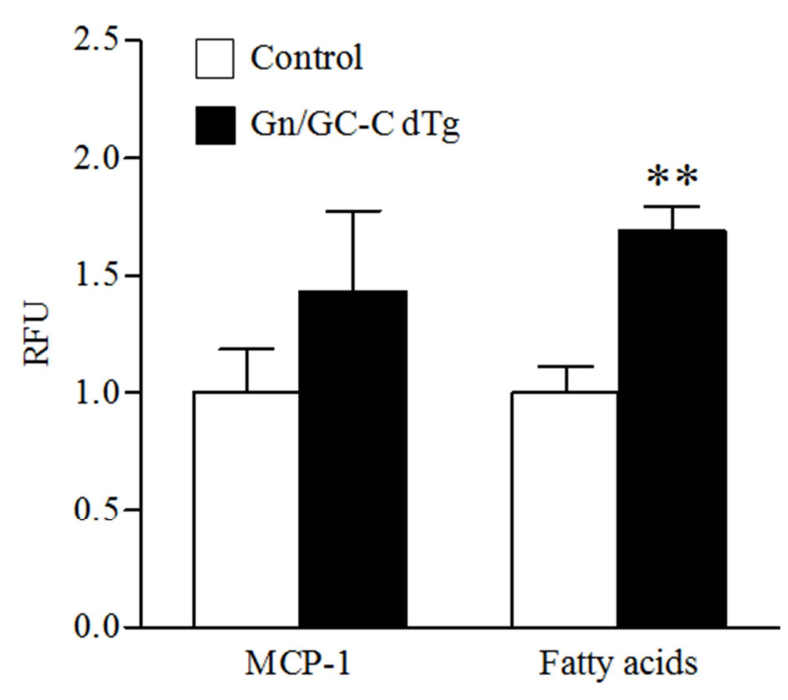

Fig. 2. Chemotaxis of peritoneal macrophages from $G n / G C-C d T g$ and wild-type (WT) rats. After 24-h induction with monocyte chemoattractant protein $(M C P) 1(M C P-1 ; 200 \mathrm{ng} / \mathrm{ml})$ or fatty acid mixture (fatty acids; 600 $\mu M)$, the chemotaxis of peritoneal macrophages was measured as relative fluorescent units (RFU). The data are presented as means $\pm S E M(n=5$ or 7 per group). **, $P<0.01$ compared with WT rats.

\section{Conclusion}

$\mathrm{Gn} / \mathrm{GC}-\mathrm{C}$ macrophages have the unique characteristics of low levels of proinflammatory cytokines and M1 macrophage 
markers in the steady state and a high migratory ability in response to fatty acids. These characteristics of Gn/GC-C macrophages might contribute to the anti-obese phenotype of Gn/GC-C Tg rats.

\section{Acknowledgements}

This work was supported by grants-in-aid for Scientific Research on Innovative Areas from the Ministry of Education, Culture, Sports, Science and Technology of Japan; and the Takeda Science Foundation. The authors thank Akane Miyashita, Yumi Aiboshi, and Tamiko Miyanaga (University of Miyazaki, Miyazaki, Japan) for their technical assistance, and Takanori Ida (University of Miyazaki, Miyazaki, Japan) for his technical help with the Flexstation Fluorometer.

\section{References}

[1] F. Ginhoux, S. Jung, Monocytes and macrophages: developmental pathways and tissue homeostasis, Nat. Rev. Immunol. 14 (2014) 392-404.

[2] C. Shi, E.G. Pamer, Monocyte recruitment during infection and inflammation, Nat. Rev. Immunol. 11 (2011) 762-774.

[3] J.M. den Haan, L. Martinez-Pomares, Macrophage heterogeneity in lymphoid tissues, 35 (2013) 541-552.

[4] S. Gordon, P.R. Taylor, Monocyte and macrophage heterogeneity, Nat. Rev. Immunol. 5 (2005) 953-964.

[5] P.J. Murray, T.A. Wynn, Protective and pathogenic functions of macrophage subsets, Nat. Rev. Immunol. 11 (2011) 723-737.

[6] F.O. Martinez, S. Gordon, The M1 and M2 paradigm of macrophage activation: time for reassessment, F1000Prime Rep. 6 (2014) 6-13.

[7] D. Tugal, X. Liao, M.K. Jain, Transcriptional control of macrophage polarization, Arterioscler. Thromb. Vasc. 33 (2013) 1135-1144.

[8] Y.C. Liu, X.B. Zou, Y.F. Chai, Y.M. Yao, Macrophage polarization in inflammatory diseases, Int. J. Biol. Sci. 10 (2014) 520-529.

[9] A. Mantovani, A. Sica, S. Sozzani, P. Allavena, A. Vecchi, M. Locati, The chemokine system in diverse forms of macrophage activation and polarization, Trends Immunol. 2 (2004) 677-686.

[10] V.H. Perry, J. Teeling, Microglia and macrophages of the central nervous system: the contribution of microglia priming and systemic inflammation to chronic neurodegeneration, Semin Immunopathol. 35 (2013) 601-612.

[11] K. Steinwede, S. Henken, J. Bohling, R. Maus, B. Ueberberg, C. Brumshagen, E.L. Brincks, T.S. Griffith, T. Welte, U.A. Maus, TNF-related apoptosis-inducing ligand (TRAIL) exerts therapeutic efficacy for the treatment of pneumococcal pneumonia in mice, J Exp Med. 209 (2012) 1937-1952.

[12] A. Waddell, R. Ahrens, K. Steinbrecher, B. Donovan, M.E. Rothenberg, A. Munitz, S.P. Hogan, Colonic eosinophilic inflammation in experimental colitis is mediated by Ly6C (high)
CCR2(+) inflammatory monocyte/macrophage-derived CCL11, J Immunol. 186 (2011) 5993-6003.

[13] S. Pestka, C.D. Krause, D. Sarkar, M.R. Walter, Y. Shi, P.B. Fisher, Interleukin-10 and related cytokines and receptors, Annu. Rev. Immunol. 22 (2004) 929-979.

[14] S.H. Wei, A. Ming-Lum, Y. Liu, D. Wallach, C.J. Ong, S.W. Chung, K.W. Moore, A.L. Mui, Proteasome-mediated proteolysis of the interleukin-10 receptor is important for signal downregulation, J. Interferon. Cytokine .Res. 26 (2006) 281-290.

[15] V. Briken, D.M. Mosser, Editorial: switching on arginase in M2 macrophages, J. Leukoc. Biol. 90 (2011) 839-841.

[16] M. Rath, I. Müller, P. Kropf, E.I. Closs, M. Munder, Metabolism via Arginase or Nitric Oxide Synthase: Two Competing Arginine Pathways in Macrophages, Front Immunol. 5 (2014) 532.

[17] T. Lucas, A. Waisman, R. Ranjan, J. Roes, T. Krieg, W. Müller, A. Roers, S.A. Eming, Differential roles of macrophages in diverse phases of skin repair, J. Immunol. 184 (2010) 964-977.

[18] P.R. Nagareddy, M. Kraakman, S.L. Masters, R.A. Stirzaker, D.J. Gorman, R.W. Grant, D. Dragoljevic, E.S. Hong, A. Abdel-Latif, S.S. Smyth, S.H. Choi, J. Korner, K.E. Bornfeldt, E.A. Fisher, V.D. Dixit, A.R. Tall, I.J. Goldberg, A.J. Murphy, Adipose tissue macrophages promote myelopoiesis and monocytosis in obesity, Cell Metab. 19 (2014) 821-835.

[19] S.P. Weisberg, D. McCann, M. Desai, M. Rosenbaum, R.L. Leibel, A.W. Ferrante Jr, Obesity is associated with macrophage accumulation in adipose tissue, J. Clin. Invest. 112 (2003) 1796-808.

[20] C.N. Lumeng, J.L. Bodzin, A.R. Saltiel, Obesity induces a phenotypic switch in adipose tissue macrophage polarization, J. Clin. Invest. 17 (2007) 175-84.

[21] X. Prieur, C.Y. Mok, V.R. Velagapudi, V. Núñez, L. Fuentes, D. Montaner, K. Ishikawa, A. Camacho, N. Barbarroja, S. O'Rahilly, J.K. Sethi, J. Dopazo, M. Orešič, M. Ricote, A. Vidal-Puig, Differential lipid partitioning between adipocytes and tissue macrophages modulates macrophage lipotoxicity and M2/M1 polarization in obese mice, Diabetes 60 (2011) 797-809.

[22] N. Arshad, S.S. Visweswariah, The multiple and enigmatic roles of guanylyl cyclase $\mathrm{C}$ in intestinal homeostasis, FEBS Lett. 586(2012) 2835-2840.

[23] S. Akieda-Asai, M. Sugiyama, T. Miyazawa, S. Koda, I. Okano, K. Senba, P.E. Poleni, Y. Hizukuri, A. Okamoto, K. Yamahara, E. Mutoh, F. Aoyama, A. Sawaguchi, M. Furuya, M. Miyazato, K. Kangawa, Y. Date, Involvement of guanylin and GC-C in rat mesenteric macrophages in resistance to a high-fat diet, J. Lipid. Res. 54 (2013) 85-96.

[24] C.D. Dumitru, J.D. Ceci, C. Tsatsanis, D. Kontoyiannis, K. Stamatakis, J.H. Lin, C. Patriotis, N.A. Jenkins, N.G. Copeland, G. Kollias, P.N. Tsichlis, TNF-alpha induction by LPS is regulated posttranscriptionally via a Tpl2/ERK-dependent pathway, Cell 103 (2000) 1071-1083.

[25] J.Y. Huh, Y.J. Park, M. Ham, J.B. Kim, Crosstalk between adipocytes and immune cells in adipose tissue inflammation and metabolic dysregulation in obesity, Mol Cells. 37 (2014) 365-3671. 
[26] K.A. Lê, S. Mahurkar, T.L. Alderete, R.E. Hasson, T.C. Adam, J.S. Kim, E. Beale, C. Xie, A.S. Greenberg, H. Allayee, M.I. Goran, Subcutaneous adipose tissue macrophage infiltration is associated with hepatic and visceral fat deposition, hyperinsulinemia, and stimulation of NF- $\mathrm{kB}$ stress pathway, Diabetes. 60 (2011) 2802-2809.

[27] X. Prieur, C.Y. Mok, V.R. Velagapudi, V. Núñez, L. Fuentes, D. Montaner, K. Ishikawa, A. Camacho, N. Barbarroja, S. O'Rahilly, J.K. Sethi, J. Dopazo, M. Orešič, M. Ricote, A. Vidal-Puig, Differential lipid partitioning between adipocytes and tissue macrophages modulates macrophage lipotoxicity and M2/M1 polarization in obese mice, Diabetes. 60 (2011) 797-809.

[28] S.E. Shoelson, L. Herrero, A. Naaz, Obesity, inflammation, and insulin resistance, Gastroenterology, 132 (2007) 2169-2180.

[29] S.L. Deshmane, S. Kremlev, S. Amini, B.E. Sawaya, Monocyte chemoattractant protein-1 (MCP-1): an overview, J. Interferon. Cytokine. Res. 29 (2009) 313-326.
[30] D. Patsouris, J.G. Neels, W. Fan, P.P. Li, M.T. Nguyen, J.M. Olefsky, Glucocorticoids and thiazolidinediones interfere with adipocyte-mediated macrophage chemotaxis and recruitment. J Biol Chem. 284 (2009) 31223-312235.

[31] C. Yeop Han, A.Y. Kargi, M. Omer, C.K. Chan, M. Wabitsch, K.D. O'Brien, T.N. Wight, A. Chait, Differential effect of saturated and unsaturated free fatty acids on the generation of monocyte adhesion and chemotactic factors by adipocytes: dissociation of adipocyte hypertrophy from inflammation, Diabetes 59 (2010) 386-396.

[32] K. Takahashi, S. Mizuarai, H. Araki, S. Mashiko, A. Ishihara, A. Kanatani, H. Itadani, H. Kotani, Adiposity elevates plasma MCP-1 levels leading to the increased CD11b-positive monocytes in mice, J. Biol. Chem. 278 (2003) 46654-4660.

[33] S. Tateya, Y. Tamori, T. Kawaguchi, H. Kanda, M. Kasuga, An increase in the circulating concentration of monocyte chemoattractant protein-1 elicits systemic insulin resistance irrespective of adipose tissue inflammation in mice, Endocrinology. 151 (2010) 971-979. 